九州大学学術情報リポジトリ

Kyushu University Institutional Repository

\title{
Regional Hazard Prediction of Rock Bursts using Microseismic Energy Attenuation Tomography in Deep Mining
}

\section{Zhang, Mingwe i}

State Key Laboratory for Geomechanics and Deep Underground Engineering, School of Mechanics and Civil Engineering, China University of Mining and Technology

Liu, Shengdong

State Key Laboratory for Geomechanics and Deep Underground Engineering, School of Mechanics and Civil Engineering, China University of Mining and Technology

Shimada, Hideki

Department of Earth Resources Engineering, Faculty of Engineering, Kyushu University : Professor

http://hdl. handle. net/2324/4110737

出版情報：Natural Hazards. 93，pp.1359-1378，2018-06-04. Springer バージョン：

権利関係 : 


\section{Regional Hazard Prediction of Rock Bursts using Microseismic Energy Attenuation Tomography in Deep Mining}

Mingwei Zhang ${ }^{\text {a,*}}$, Shengdong Liu ${ }^{\text {a }}$, Hideki Shimada ${ }^{b}$

a State Key Laboratory for Geomechanics and Deep Underground Engineering, School of Mechanics and Civil Engineering, China University of Mining and Technology, Xuzhou, Jiangsu 221116, China

b Department of Earth Resources Engineering, Faculty of Engineering, Kyushu University, 744 Motooka, Nishi-ku, Fukuoka, 819-0395, Japan

Corresponding author: Mingwei Zhang

E-mail address: mingweizhang@cumt.edu.cn 
Abstract: Rock burst prediction is a worldwide challenge that we have long tried to overcome. This study tentatively proposed a method to regionally predict rock burst hazards using microseismic energy attenuation. To verify the feasibility of the proposal, first, the mechanism of microseismic energy propagation and attenuation in rock medium was explored, and dominant attenuation characteristics of microseismic waves were analyzed. Second, a spatial attenuation model of microseismic energy was established, and the average energy attenuation coefficient for each wave path was defined. A 3D seismic energy attenuation inversion algorithm was put forward, and the corresponding computation matrix was developed. Third, a continuous microseismic field investigation was carried out in a deep coal mine. Seismic energy attenuation coefficient was confirmed using the calibrated focus position and energy determination. Based on data discretization processing, energy attenuation inversion and tomography, potential rock burst hazard regions were strictly zoned in mining areas. Finally, regional prediction results obtained from the microseismic energy attenuation were compared with the direct measurement results obtained from the classical drilling dust method to verify the reliability of proposed approach. It turns out that rock burst hazard regions predicted by the microseismic energy attenuation agreed well with the objective hazardous situations. Seismic energy attenuation coefficient is a significant evaluation factor that directly mirrors the inelastic performance of rock medium. Energy attenuation coefficient threshold used for determining the rock burst hazard regions was $3.0 \mathrm{~km}^{-1}$. Reliability of the seismic energy attenuation inversion and tomography was closely related to the spatial distribution of microseisms in a localized region. The optimum spatial density of microseisms was $0.2 \mathrm{~m}^{-3}$. Regional rock burst prediction using microseismic energy attenuation is an effective approach for revealing potential hazardous regions in deep 
mining conditions. This approach improves the pertinence of geological hazard prevention and provides a beforehand reference for targeted hazard management.

Keywords: Deep underground mining; Rock burst; Regional hazard prediction; Microseismic energy attenuation coefficient; Attenuation inversion and tomography. 


\section{Introduction}

Rock bursts have become a major geological dynamic hazard in underground coal mines. As the direct manifestation of strong mining-induced tremor, rock bursts release large amounts of elastic strain energy accumulated in hard coal and rock mass, leading to serious damage and particularly threatening miners' lives. Destruction resulting from rock bursts is shown in Figure 1. Because of the serious consequences, rock bursts have become one of the most intractable problems in deep mining (Ortlepp \& Stacey 1994). Understanding how to accurately predict rock burst hazards and then effectively eradicate the events at the germination stage is a difficult challenge that we have long tried to overcome.

As the essential interlink between rock bursts and microseisms, microseismic method has been gradually applied to rock burst prediction in recent decades (Senfaute et al. 1997; Fujii et al. 1997). Microseism is a faint short-duration earth tremor induced by the dynamic instability of rock mass. It is similar to a serious explosion or light earthquake accompanied by a tremendous and toneless sound. The generating conditions and manifesting forms of microseisms are accidental, complicated and unexpected. No obvious macroscopic premonition appears before microseisms, which makes the essential elements - time of occurrence, spatial location, released energy, acting direction and internal effect on the rock structure unpredictable and uncontrollable (Grechka et al. 2011). In particular, energy released from microseisms is diverse, and corresponding intensity varies largely. Microseismology has become popular in many fields. Based on spatial, remote, uninterrupted and real-time monitoring of microseisms in mining regions, factors of captured microseisms, especially the focus position and released energy, imply the mode, range, location and direction of rock failure and stress drop on a spatial scale, 
which helps in the prediction of rock burst hazards (Sharan 2007). This quality makes microseismic method an important and advanced measure for the timely and effective management of geological disasters. Microseismic method plays an indispensable role in safe mining in deep mines. As Ge (2005) noted, microseismic technique has grown from a pure research means for studying rock burst to a basic industrial tool for daily safety monitoring at rock burst-prone mines over the past 20 years.

Aiming to the microseismic application in the management of geological dynamic hazards, one of the emerging research fronts is rock burst prediction using microseismology. Relevant research interests mainly focus on three aspects. The first is microseismicity. Hazard prewarning models within a short period are built based on the exploration of spatial and temporal evolution of microseisms with mining activities. Rock burst precursors are tried to reveal combining with the geologic tectonic features in working field. Using real-time microseismic data and an established rock burst warning formula, Feng et al. (2015) proposed a microseismicity-based method of rock burst warning in tunnels to dynamically warn of and reduce the risk of rock bursts. Based on a digital multichannel microseismic monitoring, Yu et al. (2015) investigated the rock burst seismogenic processes in deeply buried tunnels and provided a theoretical basis for their prediction. Zhang et al. (2013) proposed a method for rock burst hazard assessment in mines on the basis of a long-term, large-scale microseismicity observation. Based on improved microseismic data, Chen et al. (2013) analyzed and predicted rock burst hazards during boring machine excavation in tunnels covered by an approximately $2000 \mathrm{~m}$ thick rock mass. Using simple but effective analysis techniques of high-resolution microseismic data, Hudyma and Potvin (2010) described an engineering approach to seismic risk management in hardrock mines. 
The second aspect is the joint time-frequency analysis of microseismic waves. A comparison of the time-frequency characteristics between rock burst signals and microseismic signals is implemented to explore the same or similar features in time domain and frequency spectrum of these two types of waves. This process helps to forecast the potential upcoming rock bursts by means of capturing similar features in continuously emerging microseisms. Based on rock burst evaluation and forecasting in deep coal mines on the basis of microseismic monitoring, Lu et al. (2012) analyzed the frequency-spectrum evolution in detail using the band-stop filter and Fourier transform to explore the microseismic low-frequency precursor effect of bursting failure in coal and rock mass. Above research interests are common and applied widely.

The third aspect is microseismic inversion and imaging, which involves the most recent and cutting edge research. Elastic energy released from a microseismic focus propagates in the form of waves and attenuates gradually in coal and rock mass. With the aid of geophysical theory, methods and techniques, microseismic inversion and imaging have been gradually applied to rock burst prediction (Young \& Maxwell 1992; Miao et al. 2012). Based on microseismic events recorded during a mining operation, Cao et al. (2016) presented passive tomographic imaging to assess strong tremor hazards and locate high microseismic activity zones around an island coal face under super-thick strata. Using the combination of tomography and microseismic monitoring system during the mining process, Cai et al. (2016) conducted passive microseismic velocity tomography in rock burst hazard assessment and analyzed the differences between the tomography technology and traditional methods.

Microseismic velocity inversion has attracted researchers' attention and is becoming a hot topic. By contrast, there is less research on microseismic attenuation 
inversion. In this research, in light of the existing problems in rock burst prediction, we systematically developed a regional prediction approach for rock burst hazards on the basis of microseismic energy attenuation. Reliability of the hazard prediction method was examined using microseismic field investigations. Methodological framework of microseismic attenuation inversion and tomography proposed. Findings of this study play an indispensable role in enriching the theoretical system of rock burst prediction and hazard control.

\section{Propagation and dissipation of seismic energy in rock medium}

\subsection{Energy attenuation mechanism of seismic wave propagation in rock medium}

A large amount of elastic strain energy is rapidly released after a microseism. Most of the energy propagates in surrounding rock mass in the form of a stress wave. As a form of energy transmission, seismic waves attenuate gradually with increasing propagation distance in deep rock. Rock is generally treated as a medium with viscoelastic, inhomogeneous and biphasic properties. Impact of the rock medium on seismic wave attenuation is significant. Seismic energy attenuation is closely related to physical properties and inner structures of the rock medium. Due to geological complexity, causes of seismic wave attenuation vary with distinct rock conditions.

Many intrinsic and extrinsic factors result in the energy attenuation. It is generally recognized that the energy attenuation effect of seismic waves is mainly caused by the elastic scattering conversion of wave front and the absorption action of rock medium (Buchanan et al. 1983). Elastic scattering loss of seismic wave energy is induced from various scales of heterogeneities of viscoelastic rock. Scattering action converts the energy of coherent, collimated waves into incoherent, divergent waves as a result of wave interaction with the inhomogeneity and discontinuity of rock medium. Energy loss in a seismic wave due to viscoelasticity combines with the loss of 
scattered energy redistributed in time and space by heterogeneity, which is the reflection loss and transmission loss at an interface, or the scattering losses at a rough surface.

Seismic energy attenuation due to rock absorption focuses on two aspects: the elastoplastic strain of rock elements and the internal friction of rock particles. For the elastoplastic strain, rock inner structure changes to some extent under a continuous external force. Rock element cannot reinstate the original state completely when the external force disappears. This faint residual strain consumes part of the wave energy. The fractional loss of seismic wave energy in rock with elastoplastic deformation lags in time, and the applied stress is associated with the wave mode in one diffusion cycle. By contrast, the internal friction in rock leads to mutual friction appearing between adjacent rock particles during the wave propagation (Carcione et al. 1988). Some portion of the wave energy is dissipated to overcome the internal friction between particles. Imperfect elasticity of rock material induces irreversible energy conversion from a mechanical state to a thermal state via viscosity, relaxation, heat conduction, elastic hysteresis, etc. Energy absorption action is an intrinsic attribute of the rock medium, which in most situations is treated as the dominant cause for seismic energy attenuation. Absorption characteristics determine the energy attenuation behavior of seismic waves.

\subsection{Dominant attenuation characteristics of seismic waves propagating in deep} rock

Intuitively, the energy attenuation behavior of seismic waves manifests as the rapid decrease of wave amplitudes. Reduction of the wave amplitude directly mirrors the intensity attenuation of a microseism. Energy attenuation behavior of seismic waves also appear as the variation of wave components in the frequency spectrum 
(Chelidze et al. 1996). Wave components in the entire frequency domain are partly absorbed by the rock medium, which is generally defined as the filtering performance of rock mass. Filtering action of the rock medium decreases the frequency richness, wave resolution and energy. With the wave component absorption in the low-frequency range, dissipation of wave components in the high-frequency range is more obvious. Amplitude and width of the dominant frequency band decrease gradually, which leads to the concentration of residual wave components in the low-frequency domain. Compared to seismic waves with the short propagation distance, seismic waves with the remote propagation distance have the briefer features in the frequency-energy spectrum. With the increase of the propagation distance, attenuation variation in the entire frequency-energy spectrum of seismic waves is compared in Figure 2.

\subsection{Mathematical representation of seismic energy attenuation}

The propagation of elastic strain energy resulting from the seismic focus induces elastic deformation and the slight vibration of rock particles near their equilibrium positions. Rock particles, as the energy carrier, are equipped with mechanical energy by seismic waves. Seismic wave transmission in the rock medium is uninterrupted. Because the wave path is remote, in ideal conditions, seismic focus is treated as a punctate source, and rock mass is perceived as the isotropic and homogeneous medium. Wave propagation is considered in the ray approximation; that is, the front of the divergent wave is a normative equiphase surface. As shown in Figure 3, when a seismic wave travels through the equiphase surface $a$ and the equiphase surface $b\left(r_{a}\right.$ $<r_{b}$ ), residual wave energy in these two equiphase surfaces is equivalent. That is, $\varepsilon\left(r_{a}\right) S\left(r_{a}\right)=\varepsilon\left(r_{b}\right) S\left(r_{b}\right)$ 
The wave duration remains the same as the propagation distance increases from $r_{a}$ to $r_{b}$. Above relation implies that the vibration amplitude of the spherical wave velocity is reciprocal to the propagation distance. However, due to the energy absorption effect of rock medium, wave duration increases but the wave amplitude decreases in actual conditions as the propagation distance increases. Absorption effect is the energy loss caused by the imperfections of rock medium in the form of energy conversion from mechanic to thermal. This loss can be accounted for by using the attenuation relation in the following form:

$$
A\left(r_{a}\right)=A\left(r_{b}\right) \sqrt{\eta\left(r_{b-a}\right)}
$$

where $\eta(r)=e^{2 \alpha r} . \alpha$ is the energy attenuation coefficient of seismic waves. Energy dissipation and attenuation in rock medium are inherent attributes of seismic waves. Energy attenuation coefficient is applied to reflect the energy absorption capability of the propagation medium. Because wave energy attenuation is caused by the imperfection of rock mass, an apparently larger magnitude means that more cycles are needed to thoroughly dissipate the wave energy. In this case, the propagation medium tends to be more perfect or more elastic. Absorption effect of the rock medium can be treated as a low-pass filter when energy attenuation coefficient is constant.

\section{Matrix system of spatial seismic energy attenuation inversion and tomography}

\subsection{Determination of the average energy attenuation coefficient for each wave} path

Compared to active seismic exploration, focus location $\left(x_{0}, y_{0}, z_{0}\right)$, released energy $\left(E_{0}\right)$ and origin time $\left(t_{0}\right)$ of a passive seismic event such as the microseism are unknown. Only the location of wave detectors and the arrival time of seismic waves can be measured. Under these circumstances, the determination of key elements including the origin time, focus location and released energy, is of primary 
importance. Focus location is confirmed using the calibrated positioning, and wave propagation distance between the focus and the detector is obtained. Meanwhile, residual wave energy at a specific location is achieved based on the monitored seismic waves. As Formula (2) indicates, seismic focus energy can be computed using the potential residual energy at a specific propagation distance. Focus energy determination model is shown in Figure 4. The Napierian logarithmic transformation in Formula (2) is indispensable and is processed as follows:

$\ln (E)=-2 \alpha R+\ln \left(E_{0}\right)$

Magnitude of the Napierian logarithm of residual energy is linearly dependent on the propagation distance. Focus energy is naturally obtained by the intercept of fitting straight, which is defined as follows:

$E_{0}=e^{\left.B\right|_{R=0}}$.

Upon obtaining the focus energy and residual energy, the average energy attenuation coefficient for each wave path is obtained as follows:

$\bar{\alpha}_{n}=\frac{1}{2 R_{n}} \ln \frac{E_{0}}{E_{n}}$.

\subsection{Computation matrix of $3 \mathrm{D}$ seismic energy attenuation inversion and}

\section{tomography}

Based on the determined average energy attenuation coefficient, confirmation of the spatial distribution of energy attenuation coefficients in each unknown mining region is essential. Inversion and tomography model of seismic energy attenuation is established as shown in Figure 5. Here, if the nth wave path is divided into $\mathrm{m}$ segments by $3 \mathrm{D}$ grids in spatial region, magnitude of the average energy attenuation coefficient for the nth wave path $\bar{\alpha}_{n}$ is defined by the following: 
$\sum_{x=1}^{u} \sum_{y=1}^{v} \sum_{z=1}^{w}\left(\overline{\bar{\alpha}}+e_{x y z}\right) \gamma_{x y z}^{n}=\bar{\alpha}_{n}$,

where $\overline{\bar{\alpha}}$ represents the overall average energy attenuation coefficient in mining regions, $e_{x y z}$ is the error of the energy attenuation coefficient in a unit cube at specific position, and $\gamma$ is the weight factor of the energy attenuation coefficient in corresponding unit cube, which is defined by the following:

$\gamma_{i}^{n}=\frac{l_{i}^{n}}{l_{1}^{n}+l_{2}^{n}+l_{3}^{n}+\cdots+l_{m}^{n}}, \quad i=1,2,3, \ldots, m$,

where, $l_{i}^{n}$ is the length of a part of the wave path in a specific unit cube. Segment amount $m$ for each wave path is different, which depends on the spatial location of wave detectors and microseisms. For all of the wave paths, magnitude of the average energy attenuation coefficient is determined by the static equation system:

$$
\left\{\begin{array}{l}
\sum_{x=1}^{u} \sum_{y=1}^{v} \sum_{z=1}^{w}\left(\overline{\bar{\alpha}}+e_{x y z}\right) \gamma_{x y z}^{1}=\bar{\alpha}_{1} \\
\sum_{x=1}^{u} \sum_{y=1}^{v} \sum_{z=1}^{w}\left(\overline{\bar{\alpha}}+e_{x y z}\right) \gamma_{x y z}^{2}=\bar{\alpha}_{2} \\
\cdots \\
\sum_{x=1}^{u} \sum_{y=1}^{v} \sum_{z=1}^{w}\left(\overline{\bar{\alpha}}+e_{x y z}\right) \gamma_{x y z}^{n}=\bar{\alpha}_{n} \\
\cdots \\
\sum_{x=1}^{u} \sum_{y=1}^{v} \sum_{z=1}^{w}\left(\overline{\bar{\alpha}}+e_{x y z}\right) \gamma_{x y z}^{k}=\bar{\alpha}_{k}
\end{array}, n=1,2,3, \ldots, k .\right.
$$

Above equation system is conceptually represented by the matrix equation $\mathrm{A} \Gamma=\overline{\mathrm{A}}$, 
where $\quad \mathrm{A}=\left[\begin{array}{cccc}\overline{\bar{\alpha}}+e_{111} & \overline{\bar{\alpha}}+e_{112} & \cdots & \overline{\bar{\alpha}}+e_{u v w} \\ \overline{\bar{\alpha}}+e_{111} & \overline{\bar{\alpha}}+e_{112} & \cdots & \overline{\bar{\alpha}}+e_{u v w} \\ \mathrm{M} & \mathrm{M} & \mathrm{M} & \mathrm{M} \\ \overline{\bar{\alpha}}+e_{111} & \overline{\bar{\alpha}}+e_{112} & \cdots & \overline{\bar{\alpha}}+e_{u v w}\end{array}\right], \quad \Gamma=\left[\begin{array}{cccc}\gamma_{111}^{1} & \gamma_{112}^{1} & \cdots & \gamma_{u v w}^{1} \\ \gamma_{111}^{2} & \gamma_{112}^{2} & \cdots & \gamma_{u v w}^{2} \\ \mathrm{M} & \mathrm{M} & \mathrm{M} & \mathrm{M} \\ \gamma_{111}^{k} & \gamma_{112}^{k} & \cdots & \gamma_{u v w}^{k}\end{array}\right], \quad$ and $\overline{\mathrm{A}}=\left[\begin{array}{c}\bar{\alpha}_{1} \\ \bar{\alpha}_{2} \\ \mathrm{M} \\ \bar{\alpha}_{k}\end{array}\right]$

In the given conditions, $k$ is the amount of total wave paths. Its magnitude is restricted by the product of focus amount and detector amount and determines the equation number in the matrix system. For the parametric matrix A, actual amount of the individual energy attenuation coefficient is generally determined by the unit scale of spatial grids. The amount of individual energy attenuation coefficient increases with a decrease in unit scale. The unit scale is influenced by the path amount. In most cases, the amount of unknown energy attenuation coefficient mismatches the number of matrix equations. Thus, global optimization algorithm is applied in the matrix operation.

\section{Field Investigation}

\subsection{Big data acquisition of microseisms in underground coal mine}

To verify the feasibility of the regional hazard prediction of rock bursts using microseismic attenuation inversion and tomography, a continuous microseismic observation was carried out in the Xingcun deep coal mine, which was located in east China. Instrument used in this field experiment was a seismic observation system. Primary components consisted of wave detectors, communication cable, signal acquisition device, microseismic recorder and post-processing system, which was shown in Figure 6. Through continuous monitoring, a total of 358 effective microseismic events were monitored. Arrangement of applied wave detectors and 
standard spatial distribution of monitored microseisms in the mining area were shown in Figure 7. In addition, original waves of a sampling microseism in each measuring channel were shown in Figure 8.

\subsection{Establishment of a 3D wave path model and discretization processing of microseismic attenuation coefficient}

To achieve the reliable regional prediction of rock burst hazards, focus location and focus energy of microseisms are two core factors that must be determined beforehand as accurately as possible. In this study, microseismic focus location and energy magnitude were calibrated by focus repositioning and energy redetermination. Because the actual meandering wave path was intangible and could not be known, the applied wave paths were equivalent to straight during the remote propagation. On the basis of specific spatial position of microseisms and wave detectors, the wave path model in three directions was established as shown in Figure 9, which showed that the spatial density of wave paths depended greatly on the spatial distribution of microseisms and detectors.

After the occurrence of microseisms, the entire underground country rock was passed through by large quantities of microseismic waves. The high-density microseismic energy attenuation coefficients in different directions were obtained from distinct wave paths. Using discretization processing on the energy attenuation coefficient for each wave path, the linear wave path was full of separate energy attenuation coefficients. Thus, the distribution of energy attenuation coefficients throughout the mine was varied and complicated, changing greatly in different spatial zones. The average spatial density of energy attenuation coefficients in unit cube indicated the comprehensive energy attenuation capability of microseismic waves in a regional rock mass. To visualize the energy attenuation coefficients, the data were 
expressed by numerous spheres with distinct sizes, as shown in Figure 10, which also showed the corresponding three-dimensional views. Spatial distribution of these energy attenuation coefficients was directly mirrored by the spatial density of spheres. The larger sphere indicated the lower energy attenuation.

\subsection{Investigation results - regional prediction of rock burst hazards using microseismic attenuation inversion and tomography}

On the basis of the logical relationship among rock elastoplasticity, ground stress level, rock burst probability and energy attenuation coefficient, potential rock burst hazard regions in mining area were predicted as the projective cloud charts indicated

in Figure 11. Given the microseismic energy attenuation, red regions indicate probable low-speed energy attenuation regions in deep rock, which were the predicted rock burst hazard regions based on microseismic attenuation inversion and tomography.

To verify the accuracy and reliability of regional hazard prediction results, a practical classical rock burst detection method, the drilling dust method, was applied. Drill holes in pairs were implemented in and out of the four predicted hazard regions. Drilling locations were indicated in Figure 11. Variation of the coal dust quantity corresponding to each drill hole was compared in Figure 12. Results showed that the coal dust quantity in each hole increased slowly and equally when drilling depth was less than $6 \mathrm{~m}$. Beyond this drilling depth, coal body was in the plastic zone at the edge of the working face or fractured zone surrounding the mining roadway. In-situ stress around this zone was dispersive and low. However, as the drilling depth increased, heterization of the coal dust quantity became more serious and could be divided into three types. Coal dust quantity in drill holes b2 and c2 increased slowly. When the drilling depth exceeded $10 \mathrm{~m}$, coal dust quantity decreased slightly and tended to 
steady, which implied that the in-situ stress in drilling positions was dispersive and the level of rock burst hazard was very low. Safe mining could be performed continuously. Coal dust quantity in drill holes a 2 and $\mathrm{d} 2$ increased rapidly and fluctuated obviously. The dynamic phenomena were serious. Coal dust quantity was irregular at the alert level after $10 \mathrm{~m}$, which implied that the rock burst hazard in drill hole $\mathrm{d} 2$ was moderate but above average. Coal dust quantity in drill holes a1, b1, c1 and $\mathrm{d} 1$ increased sharply and seriously once exceeded the standard level. Drilling work could not continue after the drilling depth exceeded $6 \mathrm{~m}$ and $8 \mathrm{~m}$. The drill pipe was tightly in place and seriously stuck by the coal body. A few weak microseisms occurred. These dynamic phenomena implied that in-situ stress in these drilling positions was highly concentrated, and rock burst hazard level was high. Plastic coal body easily spewed out under the compression of high ground stress, and induced a serious rock burst.

Results of these four rock burst regions predicted using microseismic energy attenuation agreed very well with the practical situation in mining regions $\mathrm{A}, \mathrm{B}, \mathrm{C}$ and $\mathrm{D}$, which verified the reliability of microseismic attenuation inversion in application of regional rock burst prediction. In addition, other hazardous regions of rock bursts indicated by the prediction results were also clearly revealed in Figure 11, which provided significant reference for targeted hazard management in the sampling coal mine. Using the explicit partition of rock burst hazard regions, pertinence of rock burst prevention and control was improved greatly.

\section{Differences between conventional rock burst prediction methods and the proposed method}

In this study, a new approach for the regional prediction of rock burst hazards is introduced. We attempted to roughly predict rock burst hazard regions in a deep 
mining area using the microseismic energy attenuation coefficient. Compared with previous hazard prediction methods (Frid 1997; Sharan 2007; Wang \& Park 2001), there are two key differences.

On the one hand, the evaluation factor applied for rock burst prediction is the microseismic energy attenuation coefficient, which is different from the conventional velocity factor. The energy attenuation coefficient is an important parameter used to represent the attenuation characteristics of microseismic wave energy in the rock medium, which is equivalent to the exponential energy decreasing velocity on a wave path from the tremor focus to the measuring station. The energy attenuation coefficient plays a primordial role in mirroring the vibration behavior and inelastic performance of rock mass, which varies with changes in the physical and mechanical properties of rock medium. Energy attenuation coefficient is an indication of how effective a given rock medium is at energy interaction. The higher the energy attenuation coefficient magnitude is, the more likely it is that a given wave energy will dissipate in a given propagation distance. Microseismic energy attenuation coefficient for the entire wave path remains the same if rock mass is homogeneous. In reality, rock medium is anisotropic, which results in the microseismic energy attenuation coefficients vary spatially and are completely randomized. This feature makes it possible for the energy attenuation coefficient to represent the internal structures and characteristics of deep rock mass. Rock region with high or low energy absorption capability can be identified once the spatial distribution of microseismic energy attenuation coefficients is confirmed, which makes it possible to apply the coefficients in the regional hazard prediction of rock bursts in deep mining conditions.

To achieve the rough prediction of rock burst hazards using the microseismic energy attenuation coefficient, the core target is detecting the spatial energy 
absorption characteristics of deep rock mass in view of the variation of microseismic energy attenuation. Based on the spatial distribution of energy attenuation coefficients in deep rock, mining region with separate energy attenuation characteristics will ultimately be recognized. To ensure the reliability and uniformity of the microseismic focus energy, the microseismic energy is determined using the average microseismic wave attenuation characteristics. All of the wave energy is logarithmically processed. The average energy attenuation coefficient is the least square value of all of the individual energy attenuation coefficients. The specific energy attenuation coefficient in each wave path is confirmed using the wave propagation distance, microseismic focus energy and residual microseismic wave energy. The energy attenuation coefficient is needed for microseismic energy inversion and tomography. In this study, the energy attenuation coefficient threshold used for determining rock burst hazard regions was $3.0 \times 10^{-3} \mathrm{~m}^{-1}$. The adjustment of the energy attenuation coefficient threshold plays an important role in the accurate and fine presentation of hazard prediction results.

On the other hand, prediction methods for rock burst hazards are basically divided into the following two types: exact prediction and rough prediction. The hazard prediction method applied in this research belongs to the category of rough prediction. For an exact prediction, three essential elements of an unknown rock burst - occurrence time, location and released energy - are always expected to precisely figure out before its occurrence. Once these essential elements are predicted in advance, corresponding prevention measures can be implemented on the target effectively, thereby effortlessly eliminating the geological disaster. Exact prediction will play an important role in rock burst management. However, years of field operations indicate that the exact prediction of rock burst hazard is almost impossible. 
Robert J. Geller, one of the most prominent seismologists today, previously commented that natural seismic events cannot be predicted (Geller et al. 1997; Geller 1997; Geller 2011). Compared with exact hazard prediction, rough prediction of rock burst hazards has been gradually developed in mining hazard management. The ultimate aim of rough hazard prediction mainly focuses on pointing out probable regions where potential rock bursts will occur. Attention on the occurrence time and released energy of rock bursts is not essential. By exploring the hidden hazardous regions, appropriate prevention measures can be effectively implemented into these potential regions to weaken the probability of rock bursts. At present, except the velocity factor is widely applied into the rough hazard prediction, the conventional methods such as the aggregate index and multifactor coupling are also applied for the incipient hazard assessment and prediction.

In brief, a key point of this research is roughly predicting rock burst hazard regions using the microseismic energy attenuation coefficient. Rough hazard prediction is an eclectic and more practicable method for rock burst management, which achieves good results to some extent and is highly valued. Because rough prediction indicates the hidden regions of rock burst events, it improves the pertinence of geological hazard prevention and control. With regard to the challenge of rock burst prediction in deep mining, no method can achieve a totally accurate prediction (Kuksenko et al. 1987). Each method has specific adaptability and reliability. The comprehensive application of multiple methods in practice can promote objective hazard prediction from a different perspective, making their respective advantages complementary to each other, and improving the accuracy of hazard prediction as much as possible. 


\section{Solution on the regional rock burst prediction using microseismic energy attenuation}

\subsection{Relationship between rock burst probability and energy attenuation coefficient}

Probability of rock bursts in underground coal mines is closely related to the level and gradient of in-situ stress (Zhang et al. 2004). Rock burst events easily occur in a mining area with a high-stress level and stress drop. Generally, stress distribution in surrounding rock is correlated with the mechanical elastoplasticity and physical structures of country rock mass. With a high ground stress, it is easier to concentrate on rock mass with high elasticity. That is, rock burst hazard probability is relatively low in mining areas with high plasticity. For rock mass with high plasticity, because endogenic micro-fractures are richly developed, microseismic energy attenuation is quicker than that in elastic rock regions. Microseismic energy is mainly absorbed by the weak coal and rock mass. Or to say, coal and rock mass with the high plasticity tend to absorb the more microseismic energy. Thus, magnitude of the energy attenuation coefficient is proportional to the plasticity of coal and rock mass in mining area. The magnitude of energy attenuation coefficient is greater in more plastic rock regions. The elastoplasticity of country rock mass corresponds to the probability of a rock burst event. Hence, magnitude of the energy attenuation coefficient is inversely proportional to the probability of rock bursts, which can be defined as follows:

$$
f r \propto \frac{1}{\alpha}
$$

where $f r$ represents the rock burst probability in related mining regions. This logical relationship implies that the lower microseismic energy attenuation coefficient indicates the higher rock burst hazard, and vice versa.

\subsection{Effect factors on 3D spatial inversion of sampling microseisms}


When using the microseismic attenuation inversion and tomography for roughly predicting rock burst hazards, accuracy of the regional hazard prediction is closely related to the spatial density of effective microseisms. Microseismic data volume must be optimum. If the data volume is too great, there would be too much superfluous duplicate data appearing in the reserving database, which is adverse to the inversing calculation. Yet, if the data volume is too small, accuracy of the regional hazard prediction would be greatly decreased. Microseismic data volume is mainly determined by the destructive degree of deep rock mass induced by mining activities. From the perspective of time, the application experience indicates that generally the data volume obtained over two weeks is appropriate for microseismic attenuation inversion and tomography. The optimum average spatial density of microseisms is 0.2 $\mathrm{m}^{-3}$. The reliability of microseismic attenuation inversion and tomography improves with the optimization of the spatial density of effective microseisms in a localized region. In this investigation, 358 microseisms whose focus energy and location were already corrected were used as the sampling data to achieve rough rock burst prediction in deep mining regions.

These events are averagely monitored during the same period and are evenly distributed in space. Microseismic data volume is huge enough. These individuals well agree with the energy inversion requirements. Given the regional prediction of rock burst hazards is directly achieved from the inverse matrix interpolation computation of discrete microseismic energy attenuation coefficients, the optimal scale of the matrix calculation is an essential factor. The dispersion in three directions is $50 \mathrm{~m}$ when discretizing the straight wave path model.

\subsection{Impacts of geological structures and mining activities on the reliability of regional hazard prediction}


Deep geological structures are complicated, and cause serious disturbances in the reliability of hazard prediction. Identification of hazardous regions from rock elastoplasticity has a certain generality and applicability, which is mirrored by the mechanical properties and physical structures of country rock mass. Thus, reasonable determination of rock burst potential regions is necessary and important. Hazardous regions are sometimes obviously interfered with through artificial mining, for instance, goafs and roadways. These mining structures lead to a noticeable effect on microseismic wave propagation. Microseismic waves diffract when hindered by mining structures, which results in an obvious increase of energy attenuation coefficients in these particular regions. This case distorts the energy absorption features of elastoplastic rock media and leads to an adverse impact on the accuracy of regional hazard predictions of rock burst using microseismic attenuation inversion and tomography. Anomalous variations in the magnitude of energy attenuation coefficients induced by mining structures should be treated differently from the normal changes induced by the elastoplasticity and mechanical properties of rock medium.

Meanwhile, it is worth mentioning that rock burst hazard regions are not static but change gradually with mining activities. With the influence of mining activities, original structures of coal and rock mass are gradually destroyed, and the initial state of the in-situ stress distribution changes (Gay 1975). After a period of time, the spatial position of predicted hazardous regions changes to some extent, which results in a redetermined magnitude and level of rock burst hazards. Readjusted ground stress and rock structures determine the new occurrence probability of rock burst hazards in specific mining regions. Hence, periodical regional prediction of rock burst hazards on the basis of microseismic energy attenuation in a specific time interval is necessary 
for regular coal mining. Microseismic attenuation inversion and tomography in mining regions play an important role in the periodical regional prediction of rock burst hazards. Because microseismic monitoring networks cover mining areas over a wide region, a high-quality attenuation distribution can be achieved in detector-covered regions. However, wave energy attenuating characteristics should be revised based on more effective data in regions outside of the detector-covered region.

\section{Conclusions}

(1) Regional rock burst prediction using microseismic energy attenuation is an effective approach for revealing potential hazardous regions in deep mining conditions. Evaluation factor applied to regional rock burst prediction is the microseismic energy attenuation coefficient, which is used for representing the energy attenuation characteristics of microseismic waves in rock medium. Microseismic energy attenuation coefficient is quite varied and completely randomized in terms of spatial location, varying with changes in the physical and mechanical properties of rock medium. The coefficient plays a primordial role in mirroring the vibration behavior and inelastic performance of rock mass. This feature makes it possible to represent the internal structures and characteristics of deep rock. Specific energy attenuation coefficient for each wave path is confirmed using wave propagation distance, microseismic focus energy and residual microseismic wave energy. Energy attenuation coefficient threshold for determining rock burst hazard regions in the sampling coal mine was $3.0 \mathrm{~km}^{-1}$.

(2) Regional rock burst prediction using microseismic energy attenuation is directly achieved from the inverse matrix interpolation computation of discrete microseismic energy attenuation coefficients. The scale of matrix calculation is an essential factor, and the optimal dispersion in wave path is $50 \mathrm{~m}$. Reliability of 
microseismic attenuation inversion and tomography is closely related to the spatial distribution of microseisms in localized region. The optimum average spatial density of microseisms is $0.2 \mathrm{~m}^{-3}$. Microseismic data volume applied to energy attenuation inversion and tomography is related to the destruction degree of deep rock mass induced from mining activities. Generally, microseisms measured over two weeks are appropriate. These values improve the accuracy of regional hazard prediction.

(3) Regional rock burst prediction using microseismic energy attenuation is achieved in microseism-susceptible regions. Logical relationship between the probability of a rock burst event and the magnitude of the energy attenuation coefficient is inverse. The lower energy attenuation coefficient usually indicates the higher rock burst risk, and vice versa. The most obvious difference from previous methods is that rock burst hazards are regionally predicted based on the integrative inversion of the microseismic energy attenuation coefficient. This method provides a more objective and reliable result than an empirical result. Complicated geological structures cause serious disturbances in the reliability of regional hazard prediction. A reasonable determination of potential regions of rock burst hazards is necessary and important. Rock burst regions are not static, but rather change gradually with mining activities. Periodical regional prediction of rock burst hazards on the basis of the microseismic attenuation inversion is essential for regular coal mining.

\section{Acknowledgements}

Financial and general support for this research provided by the Fundamental Research Funds for the Central Universities (No. 2018ZDPY08) is gratefully acknowledged. Additionally, the authors express the sincere gratitude to the Xingcun Coal Mine for the support of microseismic field experiment. 


\section{References}

Buchanan DJ, Jackson PJ, Davis R (1983) Attenuation and anisotropy of channel waves in coal seams. Geophysics 48:133-147. doi:10.1190/1.1441453

Cai W, Dou LM, Li ZL, Gong SY, Han RJ, Liu J (2016) Verification of passive seismic velocity tomography in rock burst hazard assessment. Chinese J Geophys-CH 59:252-262. doi:10.6038/cjg20160121

Cao AY, Dou LM, Cai W, Gong SY, Liu S, Zhao Y (2016) Tomographic imaging of high seismic activities in underground island longwall face. Arab J Geosci 9:1-10. doi:10.1007/s12517-015-2087-x

Carcione JM, Kosloff D, Kosloff R (1988) Wave propagation simulation in a linear viscoelastic medium. Geophys J Int 95:597-611. doi:10.1111/j.1365-246X.1988.tb06706.x

Chelidze TL, Spetzler HA, Sobolev GA (1996) Absorption of strain waves in porous media at seismic frequencies. Pure Appl Geophys 147:25-55. doi:10.1007/BF00876435

Chen B, Feng G, Li Q, Feng X, Zhao Z (2013) Analysis of microseismic characteristic and rockburst risk during TBM excavation under deeply burried tunnel. Disaster Adv 6:122-128.

Feng GL, Feng XT, Chen BR, Xiao YX, Yu Y (2015) A microseismic method for dynamic warning of rockburst development processes in tunnels. Rock Mech Rock Eng 48:2061-2076. doi:10.1007/s00603-014-0689-3

Frid V (1997) Rockburst hazard forecast by electromagnetic radiation excited by rock fracture. Rock Mech Rock Eng 30:229-236. doi:10.1007/BF01045719 
Fujii Y, Ishijima Y, Deguchi G (1997) Prediction of coal face rockbursts and microseismicity in deep longwall coal mining. Int J Rock Mech Min 34:85-96. doi:10.1016/S1365-1609(97)80035-4

Gay NC (1975) In-situ stress measurements in Southern Africa. Tectonophysics 29:447-459. doi:10.1016/0040-1951(75)90173-0

Ge M (2005) Efficient mine microseismic monitoring. Int J Coal Geol 64:44-56. doi:10.1016/j.coal.2005.03.004

Geller RJ (1997) Earthquake prediction: a critical review. Geophys J Int 131:425-450. doi:10.1111/j.1365-246X.1997.tb06588.x

Geller RJ (2011) Shake-up time for Japanese seismology. Nature 472:407-409. doi:10.1038/nature10105

Geller RJ, Jackson DD, Kagan YY, Mulargia F (1997) Earthquakes cannot be predicted. Science 275:1616-1620. doi:10.1126/science.275.5306.1616

Grechka V, Singh P, Das I (2011) Estimation of effective anisotropy simultaneously with locations of microseismic events. Geophysics 76:143-155. doi:10.1190/geo2010-0409.1

Hudyma M, Potvin YH (2010) An engineering approach to seismic risk management in hardrock mines. Rock Mech Rock Eng 43:891-906. doi:10.1007/s00603-009-0070-0

Kuksenko VS, Inzhevatkin IE, Manzhikov BT, Stanchits SA, Tomilin NG, Frolov DI (1987) Physical and methodological principles of rock burst prediction. J Min Sci+ 23:6-17. doi:10.1007/BF02534035

Lu CP, Dou LM, Liu B, Xie YS, Liu HS (2012) Microseismic low-frequency precursor effect of bursting failure of coal and rock. J Appl Geophys 79:55-63. doi:10.1016/j.jappgeo.2011.12.013 
Miao HX, Jiang FX, Song XJ, Yang SH, Jiao JR (2012) Tomographic inversion for microseismic source parameters in mining. Appl Geophys 9:341-348. doi:10.1007/s11770-012-0347-z

Ortlepp WD, Stacey TR (1994) Rockburst mechanisms in tunnels and shafts. Tunn Undergr Sp Tech 9:59-65. doi:10.1016/0886-7798(94)90010-8

Senfaute G, Chambon C, Bigarre P, Guise Y, Josien JP (1997) Spatial distribution of mining tremors and the relationship to rockburst hazard. Pure Appl Geophys 150:451-459. doi:10.1007/978-3-0348-8814-1_6

Sharan SK (2007) A finite element perturbation method for the prediction of rockburst. Comput Struct 85:1304-1309. doi:10.1016/j.compstruc.2006.08.084

Wang JA, Park HD (2001) Comprehensive prediction of rockburst based on analysis of strain energy in rocks. Tunn Undergr Sp Tech 16:49-57. doi:10.1016/S0886-7798(01)00030-X

Young RP, Maxwell SC (1992) Seismic characterization of a highly stressed rock mass using tomographic imaging and induced seismicity. J Geophys Res 97:12361-12373. doi:10.1029/92JB00678

Yu Q, Tang CA, Li L, Cheng G, Tang LX (2015) Study on rockburst nucleation process of deep-buried tunnels based on microseismic monitoring. Shock Vib 501:685437. doi:10.1155/2015/685437

Zhang M, Shimada H, Sasaoka T, Matsui K, Dou L (2014) Evolution and effect of the stress concentration and rock failure in the deep multi-seam coal mining. Environ Earth Sci 72:629-643. doi:10.1007/s12665-013-2985-8

Zhang M, Shimada H, Sasaoka T, Matsui K, Dou L (2013) Seismic energy distribution and hazard assessment in underground coal mines using statistical 
energy analysis. Int J Rock Mech Min 64:192-200.

doi:10.1016/j.ijrmms.2013.09.001 


\section{Figure Captions}

Figure 1. Serious destruction of mining roadways and machineries induced by the rock burst occurring in a deep underground coal mine.

Figure 2. Comparison of the attenuation variation in the entire frequency-energy spectrum of microseismic waves with the increase of the propagation distance.

Figure 3. Normative analytical mathematical model of the seismic energy emission and attenuation in the rock medium.

Figure 4. Schematic diagram of the microseismic occurrence, propagation and monitoring in the spatial region.

Figure 5. Theoretical model of the $3 \mathrm{D}$ seismic energy attenuation inversion and tomography.

Figure 6. Main components of the measuring instrument for the continuous microseismic observation.

Figure 7. Spatial distribution of wave detectors and monitored seismic events in the related mining region.

Figure 8. Original waveform and essential information of a microseism in each monitoring channel.

Figure 9. Simplified wave path model of specific microseismic waves in three dimensions.

Figure 10. Visual distribution of energy attenuation coefficients in effective spatial domain and the corresponding three dimensional views.

Figure 11. Regional prediction results on rock burst hazards in the sampling coal mine using the seismic energy attenuation inversion and tomography.

Figure 12. Comparison of the coal dust quantity with the increase of the drilling depth in each drill hole. 